\title{
BERGMAN under MS-DOS and Anick's resolution
}

\author{
S. Cojocaru and V. Ufnarovski
}

Institute of Mathematics, Academy of Sciences of Moldova, 5 Academiei str., Kishinev, 277028, Moldova

E-Mail: ufn@maths.1th.se 22svet@math.moldova.su

\begin{abstract}
Noncommutative algebras, defined by the generators and relations, are considered. The definition and main results connected with the Gröbner basis, Hilbert series and Anick's resolution are formulated. Most attention is paid to universal enveloping algebras. Four main examples illustrate the main concepts and ideas. Algorithmic problems arising in the calculation of the Hilbert series are investigated. The existence of finite state automata, defining the behaviour of the Hilbert series, is discussed. The extensions of the BERGMAN package for IBM PC compatible computers are described. A table is provided permitting a comparison of the effectiveness of the calculations in BERGMAN with the other systems.
\end{abstract}

Keywords: Gröbner basis, Hilbert series, resolution

\section{Introduction}

BERGMAN is an effective program for calculating the Gröbner basis (both for commutative and noncommutative case) and monomial Poincaré series, elaborated at Stockholm University (J. Backelin) for SUN-station (and some other types of computers). In our implementation on an IBM PC, we used the original source of J. Backelin (and his valuable help) [1], and added some new additional functions, especially for the noncommutative algebras. To illustrate them we give all necessary definitions and several examples.

\section{Main Examples}

Let $A=\langle X| R>$ be a finitely presented (noncommutative) associative algebra over field $K$. Here are the main examples of algebras that will help us to illustrate some following definitions (later we will refer to them as main examples).

Example 2.1 $A=<x, y \mid x^{2}=0, x y^{2}=0>$

Example 2.2 $A=<x, y \mid x^{2}=y^{2}>$

Example 2.3 $A=\left\langle x, y \mid x^{2}-x y\right\rangle$

Example 2.4 $A=<e_{1}, e_{2}, e_{3}, \ldots \mid\left[e_{i}, e_{j}\right]=(i-j) e_{i+j}>$, where char $K=0$ and $[a, b]=a b-b a$. 
Though the last example does not look as finitely presented, it is evident that $e_{1}$ and $e_{2}$ generate the algebra $\left(e_{k+1}=\frac{\left[e_{k}, e_{1}\right]}{k-1}\right.$ for $\left.k \geq 2\right)$. Slightly less trivial is the fact that it is sufficient to have only two relations: $\left[e_{3}, e_{2}\right]=e_{5} ;\left[e_{4}, e_{3}\right]=e_{7}$ (see: Ufnarovskij [2]). Nevertheless, we will use this example in this infinite presentation to allow the reader to see how definitions work in the infinite case.

This is also an example of an universal enveloping algebra: $A=U(L)$, where $L$ is a Lie algebra with the same sets of generators and relations except that the commutator $[a, b]$ is now interpreted as a Lie product. If we interpret it as a graded commutator $[a, b]=a b-(-1)^{|a||b|} b a$, the second example can also be considered as an universal enveloping algebra of 4-dimensional Lie superalgebra: $L=<x, y \mid[x, x]=$ $[y, y]>$.

\section{Hilbert Series and Global Dimension}

Note that all algebras in our examples are graded algebras: $A=\oplus A_{n}$, where all components $A_{n}$ are finite dimensional and $A_{n} A_{m} \subseteq A_{m+n}$. For the last example grading is less trivial: $e_{n} \in A_{n}$.

We restrict our attention on graded algebras and introduce the following:

Definition 3.1 The generating function $H_{A}=H_{A}(t)=\sum_{1}^{\infty}\left(\operatorname{dim} A_{n}\right) t^{n}$ is called Hilbert series of an algebra A.

The Hilbert series of a graded algebra is one of our main objects of interest. It is a very useful invariant in the commutative case, but in the noncommutative case it also contains a lot of important information about the algebra. First, it plays the role of generalized dimension of an algebra. For example, it has the following trivial properties:

- $H_{A \oplus B}=H_{A}+H_{B} ; H_{A \otimes B}=H_{A} H_{B}, \frac{1}{H_{A * B}}=\frac{1}{H_{A}}+\frac{1}{H_{B}}-1$.

- If $L$ is a graded Lie algebra (superalgebra), $H_{L}=\sum_{1}^{\infty} a_{n} t^{n}$ and $A=U(L)$, then

$$
H_{A}=\prod_{1}^{\infty} \frac{1}{\left(1-t^{n}\right)^{a^{n}}}\left(H_{A}=\prod_{1}^{\infty} \frac{\left(1+t^{2 n-1}\right)^{a_{2 n-1}}}{\left(1-t^{2 n}\right)^{a_{2 n}}}\right) .
$$

If $L$ is a Lie algebra from our main Example 4, then

$$
H_{L}=t+t^{2}+t^{3}+\cdots \Rightarrow H_{A}=\prod_{1}^{\infty} \frac{1}{1-t^{n}}=\sum p(n) t^{n}
$$

where $p(n)$ is the number of partitions. So it is the example with non-rational Hilbert series.

In the second main example we have $H_{L}=2 t+2 t^{2} \Rightarrow H_{A}=\frac{(1+t)^{2}}{\left(1-t^{2}\right)^{2}}=\frac{1}{1-2 t+t^{2}}$.

In our main Examples 1 and 3, the Hilbert series is equal to $\left(1-2 t+t^{2}\right)^{-1}$. It can be directly checked from the first example, but the last one will be discussed in the sequel.

Secondly, the following two theorems of Anick [3, 4] shows non-trivial properties of Hilbert series:

Theorem 3.1 Let $H_{X}\left(H_{R}\right)$ be the generating function of the number of the generators (relations, both minimal) of given degree. Then

$$
\text { gl. } \operatorname{dim} A \leq 2 \Leftrightarrow H_{A}=\left(1-H_{X}+H_{R}\right)^{-1}
$$

(inequality for free algebras only). 
In our main examples the value of $\left(1-H_{X}+H_{R}\right)$ is equal to

$$
\begin{array}{c|c|c}
1 & 2,3 & 4 \\
\hline 1-2 t+t^{2}+t^{3} & 1-2 t+t^{2} & 1-t-t^{2}+t^{5}+t^{7}
\end{array}
$$

so only the second and third examples are algebras of global dimension 2 .

Theorem 3.2 For every system of diophantine equations $S=0$, there exists a finitely presented algebra $A$ (which can be constructively expressed in terms of the coefficients of the $S$ ) such that A has global dimension 2 if and only if the system $S=0$ has no solutions.

Moreover, this algebra is an universal enveloping algebra of a Lie superalgebra, defined by quadratic relations only. This theorem is important.

\section{Corollary 3.1 One cannot}

- Find an algorithm that takes relations as input and gives the Hilbert series as output. (Furthermore, one cannot detect in general if the Hilbert series of a given algebra is equal to some fixed series.)

- Predict the behaviour of a Hilbert series, knowing only a finite number of its coefficients.

\section{Normal Words and Gröbner Basis}

Despite pessimistic conclusions in the end of the previous section, it is possible to find the Hilbert series in important cases. Let us introduce some important definitions.

Let $S$ be the set of all the words in the alphabet $X$ (identifying 1 with the empty word). Consider the following ordering on $S: f>g$ if either the length of word $f$ is greater than that of $g$ or they have the same length, but $f$ is greater then $g$ lexicographically. (More ingenious, the so-called admissible ordering may be considered too, but we restrict our attention only to this case.)

Definition 4.1 A word $f \in S$ is called normal (for $A$ ) if it cannot be written in $A$ as a linear combination of words that are less than $f$.

In our first main example the words $1, x, y, x y, y x, y^{2}$ are normal, but $x^{2}, x y^{2}$ are not. The same is true for the second main example. Why is $x y^{2}$ not normal? Because $x y^{2}=x^{3}=y^{2} x$.

In the last main example the words of the form $e_{1}^{k_{1}} e_{2}^{k_{2}} \cdots e_{m}^{k_{m}}$ are normal according the PoincaréBirkgoff-Witt-theorem, if the alphabet and ordering is $e_{1}<e_{2}<e_{3} \cdots$. It is much more complicated to express normal words in the alphabet $e_{1}, e_{2}$ only.

The following evident theorem explains how normal words can be used for the calculation of the Hilbert series.

Theorem 4.1 The set $N$, consisting of all normal words, forms a basis for the algebra A. Its Hilbert series can be calculated as $H_{A}=\sum_{0}^{\infty} d_{n} t^{n}$, where $d_{n}$ is the number of normal words of degree $n$.

Following Anick, let us introduce

Definition 4.2 A word $f \in S$ is called an obstruction if $f$ is not normal itself, but every proper subword is normal. 
Note that other expressions (such as 'tips', for example) are used instead of 'obstruction'. We denote the set of all obstructions as $F$. Because obstructions are not normal words, every $f_{i} \in F$ can be written as a linear combination of normal words: $f_{i}=u_{i}$.

Definition 4.3 The set $G=\left\{f_{i}-u_{i}\right\}$ is called a (reduced) Gröbner basis (for A).

In our main examples:

1. $G=\left\{x^{2}, x y^{2}\right\}$ (evidently).

2. $G=\left\{x^{2}-y^{2}, x y^{2}-y^{2} x\right\}$.

3. $G=\left\{x y^{k}-x y^{k+1} \mid k=0,1,2, \ldots\right\}$.

4. $G=\left\{e_{j} e_{i}-e_{i} e_{j}-(j-i) e_{i+j} \mid j>i\right\}$.

All those examples can be easily checked by hand (see [5]). BERGMAN is a powerful tool to calculate Gröbner basis in the more complicated cases.

Of course, knowing an obstruction set we can reconstruct normal words:

Theorem 4.2 Let $B=\langle X \mid F\rangle$. Then algebras $A$ and $B$ have the same sets of normal words and, in particular, $H_{A}=H_{B}$.

Definition 4.4 Algebra $B$ from the previous theorem is called the monomial algebra, associated with $A$.

So, the algebra from our first main example is associated with the monomial algebra for our second example. Note also that all universal enveloping algebras for Lie algebras with the same Hilbert series have, according to the PBW-theorem, the same associated monomial algebra (in some alphabets). Note also that definitions depend upon choice of the generator set (alphabet) and ordering.

More generally, a Gröbner basis for any ideal $I$ is its subset $G$, such that the set of highest terms of elements from $G$ contains the set of obstructions for $A=K\langle X\rangle / I$. Note that the reduced Gröbner basis may be easily obtained from an arbitrary Gröbner basis (by self-reducing) and determined uniquely for a given ordering.

\section{$5 n$-chains and Poincaré Series}

The next step is to introduce some homological algebra. Let us consider a graph $\Gamma=(V, E)$, where the set of vertex $V$ consists of the union of the unit 1, alphabet $X$ and all proper suffixes of the obstructions. Edges $E$ are defined as follows: $1 \rightarrow x$ for every $x \in X$ and in other cases $f \rightarrow g$ if and only if the word $f g$ contains the only obstruction and this obstruction is its suffix (maybe coinciding with $f g$ ).

In our main examples 1 and 2 , the graph $\Gamma$ looks like

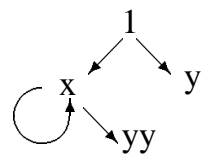

In the third example, vertices (except 1 and $y$ ) have the form $y^{n} x$ and are connected to each other (including itself).

It the fourth main example, vertices are $e_{i}$ and (considering 1 as $e_{\infty}$ ) every $e_{i}$ is connected with every $e_{j}$ with $i>j$. 
Definition $5.1 n$-chain is a word, that can be read in graph $\Gamma$ during a path of length $(n+1)$, starting from 1 . Let $C_{n}$ be a set of all $n$-chains.

So,

The only -1 -chain is 1 itself: $C_{-1}=1$.

The only 0-chains are letters from the alphabet: $C_{0}=X$.

The only 1-chains are obstructions: $C_{1}=F$.

Let us enumerate $n$-chains for $n \geq 2$ in our examples. In the first two

$$
C_{n}=\left\{x^{n+1}, x^{n} y^{2}\right\}
$$

In the third

$$
C_{n}=\left\{x y^{k_{1}} x y^{k_{2}} x \cdots x y^{k_{n}} x \mid k_{i} \geq 0\right\}
$$

In the last main example

$$
C_{n}=\left\{e_{i_{1}} e_{i_{2}} \ldots e_{i_{n+1}} \mid i_{1}>i_{2}>\cdots i_{n+1}\right\}
$$

Definition 5.2 The monomial Poincaré series for an algebra $A$ is defined as

$$
P_{A}^{m o n}(s, t)=\sum c_{m, n} t^{m} s^{n}
$$

where $c_{m, n}$ is the number of $(n+1)$-chains of degree $m$.

In our first two main examples

$$
P_{A}^{m o n}(s, t)=1+2 t s+\left(t^{2}+t^{3}\right) s^{2}+\left(t^{3}+t^{4}\right) s^{3}+\cdots
$$

In the third

$$
\begin{gathered}
P_{A}^{m o n}(s, t)=1+2 t s+\sum_{m \geq n \geq 2}\left(\begin{array}{c}
m-2 \\
n-2
\end{array}\right) t^{m} s^{n} \\
=1+2 t s+t^{2} s^{2}\left(\sum_{k=0}^{\infty}(s t+t)^{k}\right) s=1+2 t s+\frac{t^{2} s^{2}}{1-t-s t}
\end{gathered}
$$

In the fourth main example $c_{m, n}$ is equal to the number of partitions of $m$ to $n$ distinct summands.

Let us recall the definition of classical Poincaré series.

Definition 5.3 A double Poincaré series for an algebra A is defined as a generating function

$$
P_{A}(s, t)=\operatorname{dim}\left(\operatorname{Tor}_{n, m}^{A}(K, K)\right) t^{m} s^{n}
$$

where $\operatorname{Tor}_{n}^{A}(K, K)$ is considered as a graded module.

From the point of view of calculation of the Hilbert series, we can restrict our attention to the monomial Poincaré series:

Theorem 5.1

$$
H_{A}^{-1}=P_{A}(-1, t)=P_{A}^{\text {mon }}(-1, t)
$$

If $A$ is monomial algebra, then $P_{A}^{m o n}(s, t)=P_{A}(s, t)$, so n-chains correspond to homology of associated monomial algebra. 
In our first two main examples we have

$$
H_{A}^{-1}=1-2 t+t^{2}+t^{3}-t^{3}-t^{4}+t^{4}+t^{5}-\cdots=1-2 t+t^{2}
$$

In the third one we also have $H_{A}^{-1}=1-2 t+t^{2}$. The reader can interpret the connections between partitions that we have as a sequence in the last main example.

\section{Anick's Resolution}

To calculate the Poincaré series in the general case, we construct Anick's resolution [6]:

$$
C_{n} \otimes A \rightarrow C_{n-1} \otimes A \rightarrow \cdots C_{-1} \otimes A \rightarrow K \rightarrow 0
$$

It is sufficient to define module homomorphisms $d_{n}: C_{n} \otimes A \rightarrow C_{n-1} \otimes A$ only for terms $f \otimes 1$. It is convenient to identify $C_{n} \otimes N$ with $C_{n} N$. Then the map $d_{n}$ is defined as

$$
d_{n+1}(f)=f-i_{n} d_{n}(f)
$$

and $i_{n}: \operatorname{ker} d_{n-1} \rightarrow K C_{n} N$ is defined recursively:

$$
i_{n}(u)=\alpha \hat{u}+i_{n}\left(u-\alpha d_{n}(\hat{u})\right)
$$

where $\hat{u}$ is the highest term of $u$ and $\alpha$ is its coefficient.

Note that:

- $d_{0}$ calculate, for every non-empty word $f$, its normal form $\bar{f}, i_{0}$ acts identically.

- $d_{1}$ calculate, for any obstruction, $f-\bar{f}$, i.e. recover the element of Gröbner basis from its obstruction. To apply $d_{1}$ for arbitrary word of form $f s$ one need to be more careful: $d_{1}(f s)=R_{0}(f s-\bar{f} s)$. (in general, use the map $R_{n}: C_{n+1} N \rightarrow C_{n} N$, the fixed $n$-chain in the beginning and reduce the remaining part to normal form).

In our main examples:

1. $d_{n}(f)=f$ for $f \in C_{n}$ and $d_{n}(f s)=R_{n-1}(f s)$ in general (those formulas are valid for every monomial algebra).

2. In tensor language:

$$
\begin{gathered}
d_{2}: x^{3} \rightarrow x^{2} \otimes y-x y^{2} \otimes 1 \\
d_{2}: x^{2} y^{2} \otimes 1 \rightarrow x^{2} \otimes y^{2}-x y^{2} \otimes x
\end{gathered}
$$

\section{Finite State Automata and Lie Algebras}

The main problem in noncommutative cases is that Gröbner basis is usually infinite. However, by using finite state automata we can try to predict the infinity behaviour of our Gröbner basis, or at least the obstruction set on infinity. The main idea of this approach was described ir Ufnarovsky [7], and can be illustrated here by our third main example: having sufficient terms from the obstruction set, for example, 
$x^{2}, x y x, x y^{2} x, x y^{3} x, x y^{4} x$, one can predict the whole family: $x y^{n} x$. This kind of prediction can be formalized in terms of regular languages (or equivalently, finite state automata). Quite often, this prediction gives the correct answer that can be proved using other arguments. Nevertheless, the possibility of prediction is restricted. First, it is impossible in general cases, as we have already mentioned. Second, algebras that have a regular obstruction set also have a rational Hilbert series, and either polynomial or exponential growth. So, the Hilbert series for our fourth main example could not be predicted in this manner after a finite number of calculations in terms of only two generators $e_{1}, e_{2}$. But even in a simple class of universal enveloping algebras, these predictions are impossible, as we see:

Theorem 7.1 [8] iset $L$ be a free solvable Lie algebra of solvability length $k, U(L)$ be its universal enveloping algebra. If $k>2$, then the growth of $L$ and $U(L)$ is almost exponential (less than exponential growth $\left[2^{m}\right]$ but greater than growth $\left[2^{m^{\alpha}}\right]$ for any $\left.\alpha<1\right)$.

\section{Bergman Package under MS-DOS}

The BERGMAN package was elaborated by Backelin (Stockholm University) originally for SUN/Sparc stations, written in PSL (a dialect of LISP, used in REDUCE), it was successfully transfered to an IBM PC at the Institute of Mathematics of Moldova. It can be used both under REDUCE and (more easily) under a specially written shell (implemented by A. Colesnicov and L. Malahova).

Main possibilities:

- commutative Gröbner basis calculations in two different strategies;

- noncommutative Gröbner basis calculations;

- calculations of Hilbert series and Poincaré series of the associated monomial algebra;

- arbitrary long integer or $Z_{p}$ coefficients of relations;

- input and output both in LISP and Maple notations.

From the shell there are several separate programs - specially written for the noncommutative case that allow us (after calculating the Gröbner basis) to:

- predict the behaviour of the highest terms of the infinite Gröbner basis using its finite part;

- calculate growth and Hilbert series;

- calculate Anick's resolution and Poincaré series up to a given degree (implemented by A. Podoplelov).

To estimate the possibilities that BERGMAN gives, let us consider one example.

\section{Example 8.1}

$$
\begin{gathered}
A=<x, y, z, t \mid x x-x y-y x, x z+z x-y t-t y, \\
y z+z y, z t+t z, z z, t t>
\end{gathered}
$$


Its Hilbert series can be calculated both from the noncommutative and commutative points of view (considering the homology of the related commutative algebra - see Roos [9] for details). So one can compare this with one of the best commutative programs - MACAULAY (old version). But maybe the best results could be obtained from considering this example from the point of view of Lie superalgebras.

\begin{tabular}{|c|c|c|c|c|}
\hline \multirow[t]{2}{*}{ Deg } & Time for & Time for & Time for & Time for \\
\hline & BERGMAN & MACAULAY & MACAULAY & BERGMAN \\
\hline \multirow[t]{3}{*}{$\leq$} & Sparcserver & Sparcserver & Mac & IBM-PC \\
\hline & $40 \mathrm{MHZ}$ & $40 \mathrm{MHZ}$ & 16.7 MHZ & $25 \mathrm{MHZ}$ \\
\hline & $192 \mathrm{MB}$ & 192 MB & $8 \mathrm{MB}$ & $4 \mathrm{MB}$ \\
\hline 2 & 0.32 & $<1$ & $<1$ & 1 \\
\hline 3 & 0.37 & $<1$ & 1 & 1 \\
\hline 4 & 0.44 & $<1$ & 5 & 2 \\
\hline 5 & 0.53 & 1 & 16 & 3 \\
\hline 6 & 0.61 & 4 & 75 & 3 \\
\hline 7 & 0.73 & 35 & 535 & 4 \\
\hline 8 & 0.94 & 372 & 5020 & 5 \\
\hline 9 & 1.21 & 3416 & - & 6 \\
\hline 10 & 1.56 & 31742 & - & 8 \\
\hline 11 & 2.04 & 259647 & - & 9 \\
\hline 12 & 2.84 & - & - & 12 \\
\hline 13 & 4.47 & - & - & 18 \\
\hline 14 & 7.49 & - & - & 31 \\
\hline 15 & 13.9 & - & - & 116 \\
\hline 16 & 27.8 & - & - & 477 \\
\hline 17 & 59.3 & - & - & 2443 \\
\hline 18 & 167 & - & - & 11601 \\
\hline 19 & 411 & - & - & - \\
\hline 20 & 1061 & - & - & - \\
\hline
\end{tabular}

Sparcserver 690 MP (40 MHZ Cypress Sparc,192 MB int. memory) Macintosh SE/30 (68030. 16.67MHZ,8M) and 486SX $25 \mathrm{MHz}$ IBM PC compatible computer with $4 \mathrm{MB}$ RAM were used.

As to Anick's resolution, it can be calculated both on IBM PC and UNIX-stations (and takes, for example, about 10 minutes to calculate Poincaré series up to degree 12).

Pure commutative Gröbner basis calculations can also be performed quite quickly. For example, the well-known 6-cyclic system of equations takes from 1-3 minutes (depending on ordering), and this calculation cannot be performed on the same computer both by MAPLE and MATHEMATICA).

\section{References}

[1] Backelin, J. and Hollman, J. (1982). Calculating Gröbner bases fast. To appear. 
[2] Ufnarovskij, V. (1980). Poincaré series of graded algebras. Mat.Zametki, 27(1), 21-32. (English translation: Math. Notes 27 (1980), 12-18.)

[3] Anick, D. (1982). Non-commutative graded algebras and their Hilbert series. J. Algebra 78(1), 120140.

[4] Anick, D. (1985). Diophantine equations Hilbert series and undecidable spaces. Ann. Math., II Ser. 122, 87-112.

[5] Ufnarovski, V. (1995). Combinatorial and asymptotic methods of algebra. In Algebra-VI: Encyclopaedia of Mathematical Sciences 57, Springer-Verlag, pp. 5-196.

[6] Anick, D. (1986). On the homology of associative algebras. Trans. Am. Math. Soc. 296(2), 641-659.

[7] Ufnarovski, V. (1993). Calculations of growth and Hilbert Series by Computer. Lecture Notes in Pure \& Applied Mathematics 151, Springer-Verlag, pp. 247-256.

[8] Lichtman, A. and Ufnarovski, V. (1985). On Growth of Lie Algebras. Algebra Colloquium, 2(1), 45-49.

[9] Roos, J. E. A computer-aided study of the graded Lie algebra of a local commutative noetherian ring. J. Pure and Applied Algebra, 91 (1994). 